
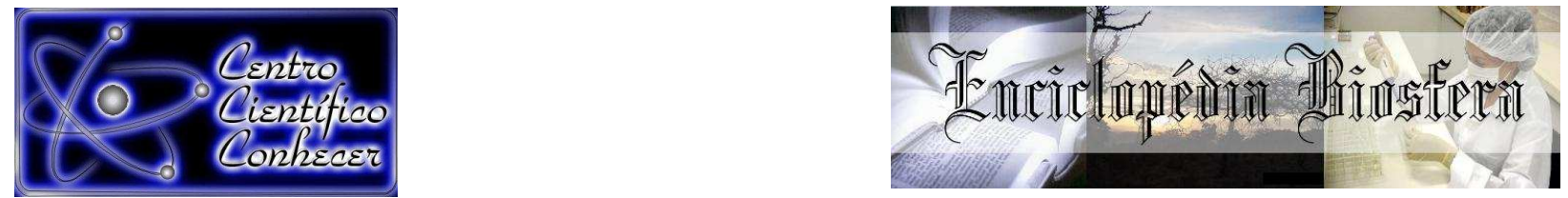

\title{
CIGARRO E NARGUILÉ: O QUE OS ACADÊMICOS PENSAM SOBRE ESSAS DROGAS?
}

Luis Fernando De Farias ${ }^{1^{*}}$, Adriana Matheus da Costa Sorato ${ }^{2}$, Valeska Marques Arruda ${ }^{2}$.

${ }^{*}$ Autor para correspondência

1. Licenciado em Ciências Biológicas, Universidade do Estado de Mato Grosso (UNEMAT), Campus Universitário de Alta Floresta. Alta Floresta - MT, Brasil. (luisfernandodefarias@gmail.com)

2. Docente da Universidade do Estado de Mato Grosso (UNEMAT), Campus Universitário de Alta Floresta. Alta Floresta - MT, Brasil.

3. Projeto ADD Saúde: Promoção e prevenção à saúde nas escolas da rede pública de Alta Floresta-MT.

Recebido em: 08/09/2015 - Aprovado em: 14/11/2015 - Publicado em: 01/12/2015 DOI: http://dx.doi.org/10.18677/Enciclopedia_Biosfera_2015_050

\section{RESUMO}

O hábito tabágico quando em grande escala pode provocar doenças consideradas graves ou letais para saúde humana. $O$ ingresso na universidade pode influenciar no convívio social e gerar situações que permitam maior vulnerabilidade para o início ao consumo de drogas como cigarro e narguilé. Assim, este estudo objetivou conhecer a percepção dos universitários em relação ao uso tabágico (cigarro e narguilé) e sugerir ações para diminuir o consumo destas drogas. Os dados foram obtidos pela aplicação de questionário a 167 estudantes da UNEMAT de Alta Floresta-MT dos cursos de Agronomia, Ciências Biológicas e Engenharia Florestal. A maioria dos estudantes foram mulheres, com 17 a 26 anos, solteiros e renda familiar de 1 a 2 salários mínimos. A maioria nunca experimentou estas drogas, e quando utilizaram o narguilé foi prevalente. Referente ao cigarro, apoiaram por ser relaxante, prazeroso. Outros, não apoiaram por viciar, provocar malefícios à saúde, por não interessar pela prática e por ser uma droga lícita. Concernente ao narguilé, os discentes apoiaram por promover prazer, bem estar, descontração, pela cultura ser interessante, por gostarem de fumar. Os entrevistados que não apoiaram o consumo afirmaram ser prejudicial, ter nojo, por ser porta de entrada a outras drogas, por viciar e por ser modismo. Dentre as ações sugeridas para diminuir o consumo destas drogas, destaca-se a realização de palestras, educação familiar e atuação dos profissionais de saúde.

PALAVRAS-CHAVE: Cachimbo D’água, Saúde coletiva, Tabagismo. 


\title{
CIGARETTE AND HOOKAH: WHAT DO ACADEMICS THINK ABOUT THESE DRUGS?
}

\begin{abstract}
The smoking habit when on a large scale can cause diseases considered serious or lethal to human health. The entry in the university can influence in the social life and create situations that allow greater vulnerability to the beginning the consumption of drugs such as cigarette and hookah. Thus, this study aimed to know the perception of university students in relation to the smoking use (cigarette and hookah) and to suggest actions to reduce consumption of these drugs. The data were collected by a questionnaire applied to 167 students from UNEMAT of Alta Floresta-MT from Agronomy, Biological Sciences and Forest Engineering courses. Most of the students were women, with 17 to 26 years old, single and family income of 1 to 2 minimum wages. The most had never experimented these drugs, and when they used the hookah was prevalent. Referring to cigarette, supported it for being relaxing, pleasurable. Others, did not support for being addictive, cause harm to health, by do not interest in practice for being a legal drug. Concerning the hookah, the students supported it to promote pleasure, wellness, relaxation, the culture be interesting, because they like to smoke. The interviewed that did not support the consumption said to be harmful, to have disgust, for being the gateway to other drugs, for being addictive and for being fad. Among the suggested actions to reduce the consumption of these drugs, stand out the realization of lectures, family education, and performance of health professionals.
\end{abstract}

KEYWORDS: Waterpipe, Public Health, Smoking.

\section{INTRODUÇÃO}

O tabagismo possui elevada morbimortalidade no mundo, e foi considerado pela Organização Mundial da Saúde (OMS) como a principal causa de morte evitável em todo o mundo (WHO, 2003). A OMS identifica a utilização de tabaco como um hábito que deve ser controlado e está associado à mortalidade para vários tipos de câncer, doença coronariana, doença pulmonar obstrutiva crônica, hipertensão arterial e acidente vascular encefálico (WHO, 2009).

Observou-se que houve queda na prevalência do tabagismo pelo cigarro, devido as campanhas contra o fumo, e as políticas públicas que foram estabelecidas nos últimos anos, que restringem o uso do cigarro. No entanto, tal restrição pode desencadear a procura de outros derivados de tabaco (BARROS \& LIMA, 2011; REVELES et al., 2013).

A entrada na universidade é um período vulnerável para o início e permanência nas drogas, pois muitos jovens entram na universidade com faixa etária e circunstâncias ideais para aquisição de novas competências, devido a isso, é preciso realizar estratégias para prevenir o uso de drogas entre a população universitária (PEUKER et al., 2006). O consumo de substâncias psicoativas entre os universitários, é um dos motivos ao uso das drogas que pode resultar em consequências negativas na vida acadêmica e prática profissional (SANTOS et al., 2013).

A vida na universidade pode gerar diversas modificações nas relações sociais e comportamentais, isso pode ocorrer em conjunto aos acontecimentos próprios da adolescência como a instabilidade psicossocial e alterações biológicas. Dessa 
maneira, o estudante torna-se mais suscetível a situações que podem oferecer risco a saúde (VIEIRA et al., 2002).

Assim, torna-se importante pesquisar sobre os fatores relacionados ao início da utilização de drogas pela juventude em nível mundial para compreender a exposição e o consumo dos jovens e auxiliar com propostas estratégicas na prevenção e tratamento (OLIVEIRA JÚNIOR et al., 2009). Portanto, objetivou-se com este estudo conhecer a percepção dos universitários da UNEMAT de Alta Floresta MT em relação ao uso tabágico (cigarro e narguilé) e sugerir ações para diminuir o consumo destas drogas.

\section{MATERIAL E MÉTODOS}

Área de estudo e população alvo

Este trabalho foi desenvolvido com os discentes de três cursos da Universidade do Estado de Mato Grosso - UNEMAT (Agronomia, Ciências Biológicas e Engenharia Florestal), que está situada na Perimetral Rogério Silva, n.4930 - Jardim Flamboyant, Campus de Alta Floresta. O município de Alta Floresta está localizado no extremo norte de Mato Grosso, com altitude média de $264 \mathrm{~m}$ e 9.310,27 km² de superfície territorial (MIRANDA \& AMORIM, 2001).

A UNEMAT foi fundada em 1978, na cidade de Cáceres-MT como Instituto de Ensino Superior de Cáceres (IESC), e foi elevada à condição de Universidade

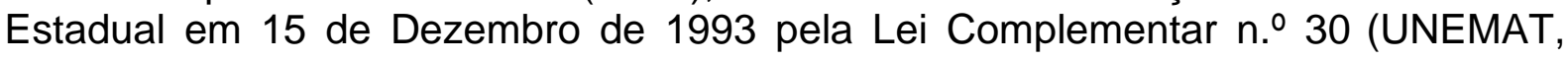
2013). O campus de Alta Floresta, teve sua criação em 1992, e atualmente oferece ensino superior para quatro cursos regulares Agronomia (Fundado em 2001), Ciências Biológicas (Fundado em 1992), Direito (Fundado em 2013), Engenharia Florestal (Fundado em 2001), e na modalidade de turma única o curso de Jornalismo (Fundado em 2013). Os cursos de Agronomia e Engenharia Florestal são de período integral e os demais cursos são noturnos.

\section{Coleta de Dados}

Neste trabalho foram entrevistados 167 estudantes matriculados nos períodos diurno e noturno, é um estudo transversal, descritivo-exploratório e quantiqualitativo. Para a coleta de dados foi utilizado um questionário investigativo, estruturado, sem identificação e auto preenchível, composto por nove questões; três discursivas e seis objetivas de uma escolha. Este instrumento de coleta de dados foi aplicado com o intuito de investigar os dados sóciodemográficos, hábito tabágico, percepção sobre as drogas e atitudes para diminuir o hábito tabágico de antigos e novos fumantes (Quadro 1).

Os questionários foram aplicados em sala de aula diretamente aos acadêmicos, após autorização do docente responsável pela disciplina, que fez uma pausa de 10 minutos da aula para aplicação desta pesquisa. Todas as informações pertinentes para 0 preenchimento dos questionários foram fornecidas aos entrevistados, ficando os mesmos livres para decidir sobre a participação na pesquisa, e foi garantida aos estudantes a confidencialidade de suas informações. 


\begin{tabular}{|c|}
\hline $\begin{array}{l}1 \text { - Questões referentes ao instrumento } \\
\text { utilizado para coleta de dados da pesquisa } \\
\text { realizada com os acadêmicos no Campus } \\
\text { de Alta Floresta, Universidade do Estado } \\
\text { de Mato Grosso (UNEMAT). }\end{array}$ \\
\hline Questões \\
\hline 1.Gênero/Sexo \\
\hline 2.Idade \\
\hline 3.Estado Civil \\
\hline 4.Renda Familiar \\
\hline $\begin{array}{l}\text { 5.Qual desses hábitos você já praticou? } \\
\text { ( ) Fumei cigarro ( ) Fumei narguilé ( ) ) Nunca fumou }\end{array}$ \\
\hline $\begin{array}{l}\text { 6. Qual dessas drogas é pior para o organismo? } \\
\text { ( ) Cigarro ( ) Narguilé ( ) As duas tem o mesmo } \\
\text { efeito }\end{array}$ \\
\hline 7.O que você pensa sobre o cigarro? \\
\hline 8.0 que você pensa sobre o narguilé? \\
\hline $\begin{array}{l}\text { 9.0 que deve ser feito para diminuir o } \\
\text { de antigos e novos fumantes de cigarro }\end{array}$ \\
\hline
\end{tabular}

FONTE: Os autores, 2015.

\section{Análise de Dados}

Os resultados obtidos foram analisados de forma quantitativa e qualitativa, os quais foram compilados com auxílio do software livre $R$ ( $R$ DEVELOPMENT CORE TEAM R, 2014) e posteriormente os dados quantitativos foram submetidos à estatística descritiva (frequência relativa, média e desvio padrão). Para comparar as proporções das respostas dos universitários utilizou-se o teste Qui-Quadrado de Pearson. Nas questões qualitativas, as respostas dos estudantes foram identificadas pela letra A (Ex: A1 - Se usado consciente não faz mal, A2 - Droga Viciante) e, as respostas semelhantes são alocadas no mesmo grupo para facilitar a discussão das mesmas (Ex. Grupo Proibição: A1- Extinção do produto; A2- Parar a Fabricação; A3Parar a produção e destruir os estocados).

\section{RESULTADOS E DISCUSSÃO}

\section{Dados Sociodemográfico e Tabagismo}

Dentre os 167 estudantes entrevistados, constatou-se que a maioria tinha idade entre 17 e 26 anos (86,23\%), renda familiar de 1 a 2 salários mínimos $(38,32 \%)$, solteiros $(85,03 \%)$ e gênero não significativo (Tabela 1$)$.

Os dados sociodemográficos (gênero, faixa etária, renda família, estado civil) podem ser fatores determinantes de risco ao uso do tabaco. Em diversos estudos constatou-se que para o cigarro, o sexo masculino apresentou predominância ao uso em relação ao feminino (ZAITUNE et al., 2012; CHKHAIDZE et al., 2013; MALTA et al., 2015). Assim como no narguilé, o sexo masculino apresenta maior frequência de uso (AL-NAGGAR \& SAGUIR, 2011; SABAHY et al., 2011; GRANT et al., 2014). 


\begin{tabular}{llll} 
TABELA 1 - Frequência relativa (\%) referente à distribuição \\
dos acadêmicos entrevistados conforme as \\
variáveis sociodemográficas no Campus de Alta \\
\multicolumn{4}{c}{$\begin{array}{l}\text { Floresta, Universidade do Estado de Mato Grosso } \\
\text { (UNEMAT). }\end{array}$} \\
\hline Categorias & Variável & Frequência (\%) & p valor* \\
\hline Gênero & Masculino & $46,11 \%$ & ns \\
Faixa Etária & Feminino & $53,89 \%$ & \\
& $17|-| 26$ & $86,23 \%$ & $<0,01$ \\
& $27|-| 36$ & $10,18 \%$ & \\
Renda Familiar & $37|-| 46$ & $1,80 \%$ & $<0,01$ \\
& $47|-| 56$ & $1,80 \%$ & \\
& Menor que 1 & $4,19 \%$ & \\
& $1-2$ salários & $38,32 \%$ & $<0,01$ \\
Estado Civil & $2-3$ salários & $26,95 \%$ & \\
& Acima de 3 & $30,54 \%$ & \\
& Solteiro & $85,03 \%$ & \\
& Casado & $13,17 \%$ & \\
& Divorciado & $1,80 \%$ & \\
&
\end{tabular}

FONTE: Os autores, 2015.

Em 2008, foi realizada uma pesquisa no Brasil e constatou-se que cerca de 25 milhões de pessoas (17,5\% da população) com 15 anos de idade ou mais utilizam produtos derivados do tabaco. Além disso, o maior percentual de fumantes ocorreu no Sul (19\%), e a menor ocorrência no Sudeste e Centro-Oeste (16,9\%). Em relação ao gênero, o masculino apresentou índice superior ao feminino em todas as regiões estudadas (BRASIL, 2009).

Nesta pesquisa a idade dos entrevistados variou de 17 a 56 anos, com média obtida foi de 22,42 $\pm 5,81$ anos. No estudo realizado por KHABOUR et al. (2012), a maioria dos entrevistados iniciaram o uso de cigarro na faixa etária entre 18 e 21 (44\%), 15 e $17(25 \%), 11$ e $14(15 \%)$ ou menos que 11 anos (10\%). Os autores também relataram que os usuários de narguilé começaram o hábito tabágico desta droga principalmente entre 18 e 21 (50\%), 15 e 17 (27\%), 11 e 14 (11\%) ou maior do que 21 anos (8\%).

Quanto à renda familiar, encontrou-se maior proporção entre 1 a 2 salários mínimos (38,32\%), seguido pelos acadêmicos com mais de 3 salários $(30,5 \%)$ (Tabela 1). Desse modo o presente estudo corrobora com as pesquisas realizadas por alguns autores que relatam existir maior frequência ao uso de cigarro entre pessoas com as menores rendas (DIAS-DAMÉ et al., 2011; OPALEYE et al., 2012; ZAITUNE et al., 2012), e no narguilé, incide em maior proporção nos usuários com maior renda familiar (AZAB et al., 2010; DUGAS et al., 2010; REVELES et al., 2013).

Em relação ao estado civil, os entrevistados em sua maioria são solteiros $(85,03 \%)$ (Tabela 1). OPALEYE et al. (2012), relatam que as pessoas que estavam separadas apresentavam maior risco de praticar o hábito tabágico de cigarro em relação aos indivíduos casados, da mesma forma, os solteiros eram mais propensos que os casados. Na pesquisa realizada por AL-NAGGAR \& SAGHIR (2011), observou-se que renda familiar e gênero foram considerados fatores de risco ao uso de narguilé, sendo que influenciaram significativamente no tabagismo desta droga, pois, os estudantes do gênero masculino e com maiores rendas apresentaram maior proporção de consumo desta droga. Entretanto, a idade, estado civil, tipo de 
faculdade, situação de vida não foram associados significativamente ao consumo de narguilé entre universitários.

Em relação ao hábito tabágico, $46 \%$ dos estudantes nunca fumaram cigarro ou narguilé, $32 \%$ experimentaram narguilé durante a vida e $22 \%$ já utilizaram cigarro (Figura 1) ( $p$ valor $<0,01$ ). Conforme estudos realizados com universitários, a predominância no consumo do cigarro como produto derivado do tabaco ocorre entre 11\% e 23\% (BOURGUIGNON et al., 2011; ALMEIDA et al., 2011; ZAITUNE et al., 2012). Outra droga que tem se destacado é o narguilé, pois em diversos países observou-se o uso em proporção de $20 \%$ a $47 \%$, e cada vez mais tem sido difundida, principalmente entre a juventude universitária (AL-NAGGAR \& SAGUIR, 2011; MARTINS et al. 2014; SALAMEH et al., 2014).

Nesta pesquisa, dos entrevistados, $62,28 \%$ acreditam que as duas drogas produzem os mesmos malefícios ao corpo humano, seguido pelo narguilé $(26,95 \%)$ e o cigarro $(10,78 \%$ ) ( $p$ valor $<0,01)$. O presente estudo observou resultado diferente do encontrado por AHMED et al. (2011), no qual os entrevistados tinham a percepção que o hábito tabágico de narguilé trazia malefícios a saúde (88\%), sendo que acreditavam que este produto era o mais prejudicial $(52 \%)$, que tanto cigarro como narguilé podem causar os mesmos danos (26\%) e tinham o pensamento que 0 cigarro causava mais prejuízos ao organismo (22\%). De acordo com MARTINS et al. (2014), é comum os usuários pensarem que utilizar narguilé não faz mal à saúde, pois acreditam ser uma forma segura de usar tabaco, pois acreditam que esta droga tem baixo risco de dependência.

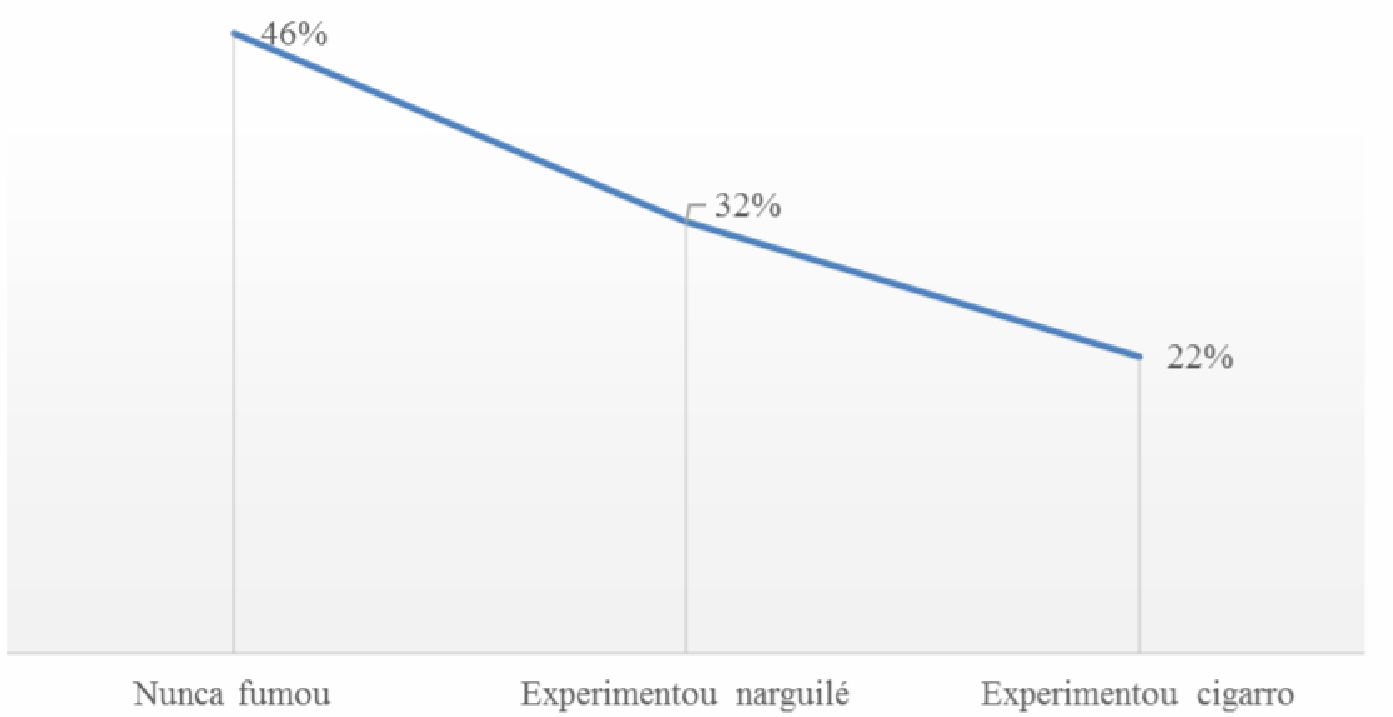

FIGURA 1 - Frequência relativa referente ao hábito tabágico de cigarro e narguilé dos acadêmicos no Campus de Alta Floresta, Universidade do Estado de Mato Grosso (UNEMAT).

FONTE: Os autores, 2015.

GALDURÓZ et al. (2007), desenvolveram uma pesquisa com estudantes brasileiros (ensino primário e secundário), entre 11 e 18 anos, que revelou queda significativa no uso de derivados do tabaco, de 32,7\% em 1997 para $25,02 \%$, em 2004, sugere-se que esta redução seja consequência das políticas públicas 
adotadas durante o período de estudo, com a proibição de publicidade do cigarro na mídia no país, assim como a inserção das advertências de fotos nas embalagens dos produtos que contém tabaco.

\section{Percepção}

Em relação à opinião dos entrevistados sobre o cigarro, houve dez respostas diferentes, as quais foram alocadas em dois grupos (Apoia ou Não apoia) para interpretar a percepção dos estudantes em relação à aceitação do uso do cigarro. Verificou-se que no grupo que Apoia, o uso do cigarro é devido ser uma atitude que provoca o relaxamento (A2) e prazerosa para quem usa, mesmo sabendo dos malefícios causados por esta droga (A1) (Quadro 2). Há trabalhos que relatam os fatores motivadores ao início da utilização desta droga, onde os mais frequentes foram a influência dos amigos, curiosidade e relaxamento (BARROS \& LIMA, 2011; BOURGUIGNON et al., 2011; FREITAS et al., 2012).

Os discentes que não apoiam o uso, tem tal pensamento devido ser uma droga que vicia, causa vários malefícios à saúde (A3, A6, A8, A9, A10), apenas por não gostar ou interessar pela prática (A4, A5). No entanto, A7 salienta que mesmo sendo uma das piores drogas existentes, mesmo assim, não foi proibida a comercialização (Quadro 2).

FERREIRA et al. (2011) observaram em seu estudo que os motivos para os não fumantes não consumirem cigarro, é porque não gostam deste hábito tabágico ou da fumaça e seu odor $(64,2 \%)$, por conhecer os prejuízos acometidos pelo consumo $(15,1 \%)$, orientação do vínculo social e de familiares $(8,8 \%)$, não apresentar vontade ou curiosidade de praticar tal hábito $(7,9 \%)$, e influência da religião $(1,3 \%)$.

QUADRO 2 - Relatos descritos pelos acadêmicos referente ao uso do cigarro no Campus de Alta Floresta, Universidade do Estado de Mato Grosso

\begin{tabular}{|l|l|l|}
\hline Categoria & Grupo & Resposta \\
\hline A1 & Apoia & Prazeroso, uma pena que prejudica a saúde. \\
\hline A2 & Apoia & Relaxante. \\
\hline A3 & Não apoia & Prejudicial à saúde, mas quem fuma acha bom. \\
\hline A4 & Não apoia & $\begin{array}{l}\text { Não tenho nada contra quem fuma, mas nunca me } \\
\text { interessei pelo hábito. }\end{array}$ \\
\hline A5 & Não apoia & Não gosto. \\
\hline A6 & Não apoia & Uma droga, causa mal à saúde. \\
\hline A7 & Não apoia & Uma das piores drogas que existem, e ainda é liberada. \\
\hline A8 & Não apoia & $\begin{array}{l}\text { Queima neurônios, nos rouba tempo e torna-se um hábito } \\
\text { vicioso. }\end{array}$ \\
\hline A9 & Não apoia & Vício horroroso, que me arrependo de ter experimentado. \\
\hline A10 & Não apoia & Causa câncer, impotência e cheira mal. \\
\hline
\end{tabular}

FONTE: Os autores, 2015.

Os estudantes foram questionados sobre o que pensam em relação ao narguilé, e as respostas descritas determinaram 11 declarações distintas, que foram separadas em dois agrupamentos (Apoia ou Não apoia) para verificar a percepção dos acadêmicos em relação à utilização deste equipamento.

Os universitários enquadrados no grupo Apoia, relataram que o narguilé produz sensação de prazer (A3), bem estar (A2), descontração (A5). Mas, A4 ENCICLOPÉDIA BIOSFERA, Centro Científico Conhecer - Goiânia, v.11 n.22; p. 33732015 
declarou utilizar apenas por achar a cultura interessante, sem sentir nada quando utiliza o artefato e A1 afirma que gosta de fumar, mesmo sabendo que é prejudicial à saúde. Estudantes pertencentes ao grupo Não apoia, citam que é prejudicial da mesma forma que o cigarro (A9), menos que o cigarro (A11), não traz benefício à saúde (A10), tem nojo (A6), droga utilizada por modismo, causa o vício a várias pessoas (A7), e porta de entrada para outras drogas (A8) (Quadro 3).

No estudo de AHMED et al. (2011) observou-se que a maioria dos entrevistados iniciaram o consumo de narguilé pela influência dos amigos e por membros da família, sendo que a maioria (88\%) acredita que o narguilé faz mal à saúde, e ainda, que esta droga é mais prejudicial do que o cigarro (52\%). Os autores também encontraram como resultado que o narguilé poderia causar diversos efeitos ao organismo (câncer, problemas de garganta, fadiga, doenças cardíacas, e dores de cabeça), e em relação ao uso, quando sentem algo após consumir narguilé, sentem tonturas, náusea, palpitações, e coração acelerado.

REVELES et al. (2013), relatam que a experimentação de qualquer tipo de produto derivado do tabaco deve ser considerada uma porta de entrada à dependência de nicotina, e assim, deve ser evitada. Também observaram que o hábito de fumar narguilé é utilizado sociavelmente, o qual permite o convívio com os amigos e outras pessoas. Esta droga apresenta estes fatores principalmente por ser utilizada por várias pessoas ao mesmo momento, com isso, percebe-se que esta droga pode ser considerada um modismo, como relata alternativa A6, pois provavelmente muitos utilizam apenas para manter este vinculo social.

QUADRO 3 - Relatos descritos pelos acadêmicos referente ao uso do narguilé no Campus de Alta Floresta, Universidade do Estado de Mato Grosso.

\begin{tabular}{|l|l|l|}
\hline Categoria & Grupo & Resposta \\
\hline A1 & Apoia & Gosto de fumar, mas sei que é prejudicial. \\
\hline A2 & Apoia & Muito bom, mas depende da essência. \\
\hline A3 & Apoia & Prazeroso. \\
\hline A4 & Apoia & $\begin{array}{l}\text { Acho cultura interessante, no entanto, não sinto nada } \\
\text { quando fumo. }\end{array}$ \\
\hline A5 & Apoia & Descontrai. \\
\hline A6 & Não apoia & Tenho nojo. \\
\hline A7 & Não apoia & Droga da moda, que está levando muitos ao vício! \\
\hline A8 & Não apoia & Porta de entrada às outras drogas. \\
\hline A9 & Não apoia & Prejudicial à saúde, tanto quanto o cigarro. \\
\hline A10 & Não apoia & Não traz benefício nenhum a saúde. \\
\hline A11 & Não apoia & Prejudicial, menos que o cigarro. \\
\hline
\end{tabular}

FONTE: Os autores, 2015.

Tem sido observado pelas pesquisas cientificas uma elevada frequência de experimentação desta droga, pois, verificou-se um indicativo da dispersão e popularidade da mesma (REVELES et al., 2013), além do que o compartilhamento da mangueira entre várias pessoas sem a esterilização necessária pode transmitir doenças, como a hepatite, herpes, resfriado, pneumonia e tuberculose.

Os acadêmicos foram questionados quanto às ações que poderiam ser realizadas para diminuir o hábito tabágico em antigos e novos fumantes de cigarro e de narguilé. Tais ações foram separadas em cinco grupos (Campanhas, Ensino, 
Legislação, Proibição, Socialização), que permitiram analisar a percepção dos estudantes em relação às propostas inseridas pelos estudantes.

Dentre as propostas sugeridas foram citadas as campanhas e palestras com o objetivo de conscientizar acerca dos prejuízos ao corpo humano (A1, A2), educação familiar (A4), e ação de médicos e psicólogos para incentivar a cessação do hábito (A3) (Quadro 4). Os resultados presentes neste estudo estão de acordo com a pesquisa realizada na Universidade de São Paulo (USP) que verificaram a opinião de estudantes de medicina, ao relatarem que há maior possibilidade dos usuários de narguilé cessarem o hábito tabágico quando são aconselhados por profissionais das ciências da saúde (MARTINS et al., 2014).

$O$ ingresso na universidade é um importante período de vida, sendo uma chance de desenvolvimento, mas também pode causar diversos riscos ao consumo de substâncias (OLIVEIRA JÚNIOR et al., 2009). Ao longo dos anos, as drogas têm sido mais frequentes no cotidiano do ambiente familiar, e também no ambiente escolar, e tem interferido nas relações sociais e pedagógicas, acarretando situações de violência física, criminalidade e agressividade. O processo de prevenção contra as drogas deve ser feito em parceria/conjunto entre escola, família, Conselho Tutelar, sociedade em geral e outros órgãos competentes. Todas as esferas da sociedade devem auxiliar nesse processo, pois as unidades escolares possuem diversos currículos obrigatórios para realizar, além de ter a função primordial de preparar o indivíduo para a vivencia em sociedade. As campanhas de prevenção às drogas devem ocorrer de forma ampla e atingir a família e sociedade como um todo e não somente por campanhas pontuais e passageiras (CABRERIZO \& IOCCA, 2014).

QUADRO 4 - Relatos descritos pelos acadêmicos referente às ações que poderiam ser feitas para diminuir o hábito tabágico em antigos e novos fumantes de cigarro e de narguilé no Campus de Alta Floresta, Universidade do Estado de Mato Grosso (UNEMAT).

\begin{tabular}{|l|l|l|}
\hline Categoria & Grupo & Ações \\
\hline A1 & Campanhas & $\begin{array}{l}\text { Campanhas de conscientização, divulgando os } \\
\text { prejuízos e malefícios do uso. }\end{array}$ \\
\hline A2 & Campanhas & $\begin{array}{l}\text { Conscientização da população, com palestras, } \\
\text { propagandas sobre os seus males, informando que } \\
\text { prejudica a saúde. }\end{array}$ \\
\hline A3 & Ensino & $\begin{array}{l}\text { Incentivar a parar o uso, com o auxílio de médicos e } \\
\text { psicólogos. }\end{array}$ \\
\hline A4 & Ensino & Educação familiar. \\
\hline A5 & Legislação & Aumentar valor do produto. \\
\hline A6 & Legislação & $\begin{array}{l}\text { Tudo que poderia ser feito já está sendo, mas muitos } \\
\text { não têm consciência. }\end{array}$ \\
\hline A7 & Proibição & Parar fabricação. \\
\hline A8 & Proibição & Parar a produção e destruir os estocados. \\
\hline A9 & Proibição & $\begin{array}{l}\text { Proibição do uso e da venda, multando quem vender, } \\
\text { sendo regulamentado por leis. }\end{array}$ \\
\hline A10 & Proibição & Proibir uso em locais públicos. \\
\hline A11 & Proibição & Extinção do produto. \\
\hline A12 & Socialização & Rejeitá-los. \\
\hline
\end{tabular}

FONTE: Os autores, 2015. 
Os discentes da UNEMAT sugerem aumentar o valor do produto (A5), tudo que poderia fazer está sendo feito (A6), parar a fabricação (A7), parar a produção, destruir todos os estoques e extinguir o produto (A8, A11), proibir usar e vender, e multar quem vender (A9) e proibir usar em locais públicos (A10) e rejeitá-los (A12) (Quadro 4).

Na legislação vigente em relação ao hábito tabágico, a Lei Antifumo (Lei no 8.262/14), que foi decretada no dia 31 de maio de 2014. Esta tem a função de proibir a utilização em lugares coletivos fechados (públicos e privados) de charutos, cigarrilhas, narguilé, cachimbos e cigarros, proibiu fumódromos, a publicidade de quaisquer produtos derivados do tabaco fumado, inclusive o narguilé. A propaganda poderá ocorrer apenas na exposição dos produtos de fumo na parte interna do local de venda e o estabelecimento comercial que vender produtos derivados do tabaco deve possuir avisos sobre os riscos e prejuízos do fumo a saúde humana, além de apresentar figuras para ilustrar as mensagens, e citar a proibição para menores de 18 anos, estabelecido pelo Estatuto da Criança e Adolescente - ECA (DIÁRIO OFICIAL DA UNIÃO, 2014).

Muitos jovens utilizam as drogas com o objetivo de obter a sensação de liberdade, saciar o prazer, esquecimento de seus medos e problemas, tanto no âmbito financeiro como psicológico e social (CABRERIZO \& IOCCA, 2014). No entanto, não é o meio adequado, pois as drogas promovem uma falsa satisfação destes desejos, mas, em contrapartida, pode resultar em sérios problemas à saúde humana, convivência social e individual. Portanto, é importante atentar que todas as formas de utilização de tabaco liberam nicotina para o Sistema Nervoso Central (SNC), promovendo o risco elevado de doença e óbito prematuro entre os usuários. Este é um produto com potencial direto ou indiretamente mortal, e o melhor é evitar a utilização deste elemento químico (VIEGAS, 2008).

\section{CONCLUSÃO}

Nesta pesquisa observou-se a maioria dos discentes acreditam que as duas drogas (cigarro e narguilé) têm o mesmo potencial de prejuízo à saúde pública. Em relação ao cigarro os entrevistados afirmaram que o uso ocorre por ser uma droga relaxante e prazerosa. Enquanto o narguilé induz ao prazer, bem estar, descontração e por ser culturalmente interessante. Assim, mesmo causando malefícios algumas pessoas preferem fumar. Mas, alguns universitários não apoiam o tabagismo por ser prejudicial à saúde, ter nojo, ser porta de entrada para outras drogas, viciar e ser uma droga da moda.

Dentre as ações propostas para diminuir o hábito tabágico em antigos e novos fumantes destaca-se a realização de palestras, educação familiar, e atuação dos profissionais da saúde. É importante o atendimento e atenção aos usuários de narguilé, pois ao longo dos anos, pesquisas científicas mostram que o uso de narguilé se sobressai em relação ao cigarro. Além disso, é necessário realizar ensaios clínicos para verificar as doenças causadas pelo narguilé, e estudos de prevalência no Brasil para entender a dependência, motivos do fumo, e fatores associados.

\section{AGRADECIMENTOS}

Os autores agradecem aos acadêmicos de Agronomia, Ciências Biológicas e Engenharia Florestal da Universidade do Estado de Mato Grosso (UNEMAT) do Campus de Alta Floresta, por auxiliarem na realização desta pesquisa. 


\section{REFERÊNCIAS}

AHMED, B.; JACOB III, P.; ALLEN, F.; BENOWITZ, N. Attitudes and Practices of Hookah Smokers in the San Francisco Bay Area. Journal of Psychoactive Drugs, v.43, n.2, p.146-152, 2011. Disponível em: < http://dx.doi.org/10.1080/02791072.2011.587707>. DOI: 10.1080/02791072.2011.587707.

ALMEIDA, J.B.; MIRANDA, J.S.; MIYASAKI, S.C.S.; MARQUES, S.F.G. Prevalência e características do tabagismo na população universitária da região de Lins - SP. Revista Brasileira de Enfermagem - REBEn, v.64, n.3, p.545-550, 2011.

AL-NAGGAR, R.A.; SAGHIR, F.S. Water Pipe (Shisha) smoking and associated factors among Malaysian university students. Asian Pacific Journal of Cancer Prevention, v.12, n.11, p.3041- 3047, 2011.

AZAB, M.; KHABOUR, O.F.; ALKARAKI, A.K.; EISSENBERG, T.; ALZOUBI, K. H.; PRIMACK, B.A.; ED, M. Waterpipe tobacco smoking among university students in Jordan. Nicotine \& Tobacco Research, v.12, n.6, p. 606-612, 2010. Disponível em:< dx.doi.org/10.1093/ntr/ntq055>. DOI: 10.1093/ntr/ntq055.

BARROS, E. R.; LIMA, R. M. Prevalência e Características do Tabagismo entre Universitários de Instituições Públicas e Privadas da Cidade de Campos dos Goytacazes, RJ. Vértices, v. 13, n. 3, p. 93-116, 2011. Disponível em: http://dx.doi.org/10.5935/1809-2667.20110027>. $\quad$ DOI: 10.5935/18092667.20110027.

BOURGUIGNON, L.N.; SILVA, B.P.; COELHO, M.P.; SIQUEIRA, M.M. O uso do tabaco entre os estudantes de enfermagem do Centro Universitário do Espírito Santo (Ceunes). Revista Brasileira de Pesquisa em Saúde, v.13, n.4, p.35-40, 2011.

BRASIL. Ministério do Orçamento e Gestão. Instituto Brasileiro de Geografia e Estatística. Pesquisa Nacional por Amostra de Domicílios: Tabagismo 2008. Rio de Janeiro: IBGE, 2009.

CABRERIZO, T.B.; IOCCA, F.A.S. Drogas no contexto escolar: processo de prevenção e sensibilização. Revista Eventos Pedagógicos, v.5, n.2, p.311-320, 2014.

CHKHAIDZE, I.; MAGLAKELIDZE, N.; MAGLAKELIDZE, T.; KHALTAEV, N. Prevalência de tabagismo e fatores que o influenciam em estudantes de medicina e outros universitários em Tbilisi, Geórgia. Jornal Brasileiro de Pneumologia, v.39, n.5, p.579-584, 2013. Disponível em:< http://dx.doi.org/10.1590/S180637132013000500008>. DOI: 10.1590/S1806-37132013000500008.

DIÁRIO OFICIAL DA UNIÃO. Decreto N.8.262 de 31 de maio de 2014. 31 mai. $2014 . \quad$ Disponível em: <http://www.planalto.gov.br/ccivil_03/_Ato20112014/2014/Decreto/D8 262.htm>. Acesso em: 24 mai. 2015. 
DIAS-DAMÉ, J.L.; CESAR, J.A.; SILVA, S.M. Tendência temporal de tabagismo em população urbana: um estudo de base populacional no Sul do Brasil. Cadernos de Saúde Pública, v.27, n.11, p.2166-2174, 2011. Disponível em: <http://dx.doi.org/10.1590/S0102-311X2011001100010>. DOI: 10.1590/S0102311 X2011001100010.

DUGAS, E.; TREMBLAY, M.; LOW, N.C.P.; COURNOYER, D.; O'LOUGHLIN, J. Water-Pipe smoking among north American youths. Pediatrics, v.125, n.6, p.11841189, 2010. Disponível em: <http://dx.doi.org/10.1542/peds.2009-2335>. DOI: 10.1542/peds.2009-2335.

FERREIRA, S.A.L.; TEIXEIRA, C.C.; CORRÊA, A.P.A.; LUCENA, A.F.; ECHER, I.C. Motivos que contribuem para indivíduos de uma escola de nível superior tornaremse ou não tabagistas. Revista Gaúcha de Enfermagem, v.32, n.2, p.287-293, 2011. Disponível em:< http://dx.doi.org/10.1590/S1983-14472011000200011>. DOl: 10.1590/S1983-14472011000200011.

FREITAS, R.L.M.; NASCIMENTO, D.S.; FREITAS, R.M.; SALDANHA, G.B.; ROCHA, R.M.M.; SANTOS, P.S. Perfil da utilização de drogas lícitas e ilícitas por universitários de uma instituição privada. Revista Eletrônica Saúde Mental Álcool e Drogas (SMAD), v.8, n.3, p.118-126, 2012.

GALDURÓZ, J.C.F.; FONSECA, A.M.; NOTO, A.R.; CARLINI, E.A. Decrease in tobacco use among Brazilian students: A possible consequence of the ban on cigarette advertising? Addictive Behaviors, v.32, n.6, p.1309-1313, 2007. Disponível em: <dx.doi.org/10.1016/j.addbeh.2006.09.004>.DOl: 10.1016/j.addbeh.2006.09.004.

GRANT, A.; MORRISON, R.; DOCKRELL, M.J. Prevalence of waterpipe (Shisha, Narghille, Hookah) use among adults in Great Britain and factors associated with waterpipe use: data from cross-sectional Online Surveys in 2012 and 2013. Nicotine \& Tobacco Research, v.16, n.7, p.931-938, 2014. Disponível em: < dx.doi.org/10.1093/ntr/ntu015>. DOI: 10.1093/ntr/ntu015.

KHABOUR, O.F.; ALZOUBI, K.H.; EISSENBERG, T.; MEHROTRA, P.; AZAB, M.; CARROLL, M.; AFIFI, R.A.; PRIMACK, B.A. Waterpipe tobacco and Cigarette among university students in Jordan. International Journal of Tuberculosis and Lung Disease (IJTLD), v.16, n.7, p.986-992, 2012. Disponível em:< dx.doi.org/10.5588/ijtld.11.0764>. DOI: 10.5588/ijtld.11.0764.

MALTA, D.C.; ANDRADE, S.S.C.A.; STOPA, S.R.; PEREIRA, C.A.; SZWARCWALD, C.L.; JÚNIOR, J.B.S.; REIS, A.A.C. Estilos de vida da população brasileira: resultados da Pesquisa Nacional de Saúde, 2013. Epidemiologia e Serviços de Saúde, v.24, n.2, p.217-226, 2015.

MARTINS, S.R.; PACELI, R.B.; BUSSACOS, M.A.; FERNANDES, F.L.A.; PRADO, G.F.; LOMBARDI, E.M.S.; TERRA-FILHO, M.; SANTOS, U.P. Experimentação de e conhecimento sobre narguilé entre estudantes de medicina de uma importante universidade do Brasil. Jornal Brasileiro de Pneumologia, v.40, n.2, p.102-110, 
2014. Disponível em: <http://dx.doi.org/10.1590/S1806-37132014000200002>. DOI: $10.1590 /$ S1806-37132014000200002.

MIRANDA, L.; AMORIM, L. Mato Grosso - Atlas Geográfico. Cuiabá: Editora Entrelinhas, 2001. 40p.

OLIVEIRA JÚNIOR, H. P.; BRANDS, B.; CUNNINGHAM, J.; STRIKE, C.; WRIGHT, M.G.M. Percepção dos estudantes universitários sobre o consumo de drogas entre seus pares no ABC paulista, São Paulo, Brasil. Revista Latino-Americana de Enfermagem, v.17, n.spe, p. 871-877, 2009. Disponível em: < http://dx.doi.org/10.1590/S0104-11692009000700018>. DOI:10.1590/S010411692009000700018.

OPALEYE, E.S.; SANCHEZ, Z.M.; MOURA, Y.G.; GALDURÓZ, J.C.F.; LOCATELLI, D.P.; NOTO, A.R. The Brazilian smoker: a survey in the largest cities of Brazil. Revista Brasileira de Psiquiatria, v.34, n.1, p.43,-51, 2012. Disponível em: < http://dx.doi.org/10.1590/S1516-44462012000100009>. DOI:10.1590/S151644462012000100009.

PEUKER, A.C.; FOGAÇA, J.; BIZARRO, L. Expectativas e beber problemático entre universitários. Psicologia: Teoria e Pesquisa, v.22, n.2, p.193-200, 2006. Disponível em: <http://dx.doi.org/10.1590/S0102-37722006000200009>. DOI: 10.1590/S0102-37722006000200009.

R DEVELOPMENT CORE TEAM. R: A language and environment for statistical computing, 2014. Disponível em <http://www.R-project.org>. Acesso em: 07 jul. 2015.

REVELES, C. C.; SEGRI, N. J.; BOTELHO, C. Factors associated with hookah use initiation among adolescents. Jornal de Pediatria, v.89, n.6, p.583-587, 2013. Disponível em: <http://dx.doi.org/10.1016/j.jpedp.2013.05.002>. DOI: 10.1016/j.jpedp.2013.05.002.

SABAHY, A.R.; DIVSALAR, K.; BAHREINIFAR, S.; MARZBAN, M.; NAKHAEE, N. Waterpipe tobacco use among Iranian university students: correlates and perceived reasons for use. The international Journal of Tuberculosis and Lung Disease, v.15, n.6, p.844-847, 2011. Disponível em: <http://dx.doi.org/10.5588/jtld.10.0744>. DOI: $10.5588 /$ ijtld.10.0744.

SALAMEH, P.; SALAMÉ, J.; WAKED, M.; BARBOUR, B.; ZEIDAN, N.; BALDI, I. Waterpipe dependence in university students and effect of normative beliefs: a crosssectional study. BMJ Open, v.4, n.2, p.1-9, 2014. Disponível em: <http://dx.doi.org/10.1136/bmjopen-2013-004378>. DOI: 10.1136/bmjopen-2013004378.

SANTOS, M.V.F.; PEREIRA, D.S.; SIQUEIRA, M.M. Uso de álcool e tabaco entre estudantes de psicologia da Universidade Federal do Espírito Santo. Jornal Brasileiro de Psiquiatria, v.62, n.1, p.22-30, 2013. Disponível em: < http://dx.doi.org/10.1590/S0047-20852013000100004>. DOI: 10.1590/S004720852013000100004. 
UNIVERSIDADE DO ESTADO DE MATO GROSSO (UNEMAT). Projeto Político Pedagógico (PPC) de graduação em Ciências Biológicas: Licenciatura Plena e Bacharelado. Alta Floresta - MT: UNEMAT, 2013.

VIEGAS, C.A.A. Formas não habituais de uso do tabaco. Jornal Brasileiro de Pneumologia, v.34, n.12, p.1069-1073, 2008. Disponível em: < http://dx.doi.org/10.1590/S1806-37132008001200013>. DOI: $\quad 10.1590 / S 1415-$ 52732002000300003.

VIEIRA, V.C.R.; PRIORE, S.E.; RIBEIRO, S.M.R.; FRANCESCHINI, S.C.C.; ALMEIDA, L.P. Perfil socioeconômico, nutricional e de saúde de adolescentes recém-ingressos em uma universidade pública brasileira. Revista de Nutrição, v.15, n.3, p.273-282, 2002. Disponível em: < http://dx.doi.org/10.1590/S141552732002000300003>. DOI: 10.1590/S1415-52732002000300003.

WORLD HEALTH ORGANIZATION (WHO). Male smoking: tobacco free initiative. Geneva: WHO, 2003.

WORLD HEALTH ORGANIZATION (WHO). WHO Report on the Global Tobacco Epidemic, 2009: Implementing smoke-free environments. Geneva: WHO, 2009.

ZAITUNE, M.P.A.; BARROS, M.B.A.; LIMA, M.G.; CÉSAR, C.L.G.; CARANDINA, L.; GOLDBAUM, M.; ALVES, M.C.G.P. Fatores associados ao tabagismo em idosos: Inquérito de Saúde no Estado de São Paulo (ISA-SP). Cadernos de Saúde Pública, v.28, n.3, p.583-595, 2012. Disponível em: <http://dx.doi.org/10.1590/S0102311X2012000300018>. DOI: 10.1590/S0102-311X2012000300018. 